\title{
Familia, escuela y adherencia a la dieta mediterránea en infancia y adolescencia
}

\section{Family, school and adherence to the mediterranean diet in childhood and}

\section{adolescence}

\author{
Natalia Abalde-Amoedo, Margarita Pino-Juste \\ Universidad de Vigo
}

\begin{abstract}
Resumen
La infancia y la adolescencia representan dos etapas de vital importancia para el desarrollo de las personas, por lo que parece imprescindible consolidar una serie de patrones que reporten beneficios a corto y largo plazo. La obesidad y el sobrepeso infantil son factores de riesgo que aumentan la probabilidad de sufrir ciertas patologías, pudiendo evitarse, en gran medida, con unos adecuados hábitos alimenticios, como la adherencia a patrones alimentarios de estilo Mediterráneo. El análisis de contenido bibliográfico de algunas de las investigaciones más recientes permite confirmar el impacto que algunos factores como familia o escuela tienen sobre la misma.

Palabras clave: entrono familiar, rol de la escuela, educación infantil temprana, adolescentes, hábitos alimenticios
\end{abstract}

\begin{abstract}
Childhood and adolescence are vital stages for people's development, so it seems essential to consolidate a series of patterns that report short and long term benefits. Childhood obesity and overweight represent risk factors that increase the probability of suffering certain pathologies and can be avoided, to a great extent, with adequate eating habits, such as adherence to Mediterranean-style eating patterns. The analysis of bibliographic content of some of the most recent researches confirms the impact that some factors, such as family or school, have on it.

Keywords: family environment, school role, early childhood education, adolescents, eating habits.
\end{abstract}

\section{Introducción}

Existe una cierta tendencia a pensar en las etapas de la vida como fases independientes, separadas las unas de las otras como compartimentos estancos. Lo cierto es que el desarrollo de los individuos es continuo a lo largo del ciclo vital, en el cual influyen factores como la herencia y el ambiente (Rice, 1997), por lo que todo aquello que ocurra en una etapa tendrá consecuencias de mayor o menor grado en la etapa siguiente. Con base en esta afirmación, y centrando el foco en las etapas de la infancia y adolescencia, parece claro que todos aquellos hábitos que se consoliden en esas edades tendrán repercusión en la última etapa, la adultez. La actividad física y la alimentación no son ajenas a esta afirmación, siendo vital instaurar los hábitos adecuados en los citados estadios (Liria, 2012; Papadaki y Mavrikaki, 2015; Roales Nieto et al., 2004; Subcomisión de Epidemiología y Comisión de Nutrición, 2005), puesto que ambos van a servir como base de sustanciación a toda la etapa adulta, incluida la vejez.

En el paso de la infancia a la adolescencia se da la mayor cantidad de aprendizajes alimentarios en el paso de la llamada "dieta láctea" a la dieta omnívora, influenciado por la predisposición genética del niño (Birch y Fisher, 1998), de ahí la importancia de prestar especial atención a las rutinas alimentarias cotidianas.

Respecto a la adolescencia, es una etapa en la que destaca el desarrollo de la identidad personal y, por otra parte, es una etapa de riesgo elevado para la adquisición de conductas nocivas para la salud (OMS, 2017), lo que obliga a un detallado control de las mismas.

La preocupación principal por esta temática no se centra en el abandono, en sí mismo, de los buenos hábitos alimenticios, sino en las repercusiones que este hecho tiene sobre el empeoramiento de la salud de la población en general.

El correcto establecimiento de patrones perdurables adecuados será vital a la hora de evitar el sobrepeso y la obesidad en el futuro dado que diversos estudios han demostrado correlaciones positivas entre ciertos datos antropométricos, por ejemplo el IMC en etapa prepuberal, y un mayor nivel de tejido adiposo en la adultez (Kindblom et al., 2009) e, incluso, un aumento del riesgo de mortalidad por patologías cardiovasculares en dicha etapa (Ohlsson, Bygdell, Sondén, Rosengren, Kindblom, 2016). Los efectos también son visibles a corto plazo, habiendo estudios que determinan que a mayores niveles de adherencia a la dieta mediterránea, menores circunferencias de cintura se hallan en adolescentes (Bacopoulou, Landis, Rentoumis, Tsitsika y Efthymiou, 2017)

Para la OMS, la obesidad es una de las epidemias del S.XXI (OMS, 2016) y algunos autores como Menéndez García y Franco Díez (2009) hablan de "obesidad epidémica", concretamente en España. En la guía elaborada por el Ministerio de Educación y Ciencia y el Ministerio de Sanidad y Consumo (2007) se apunta a un cambio de hábitos alimentarios y la reducción en el nivel de actividad física de la población como 
principales causantes del aumento de índices de obesidad y sobrepeso.

En definitiva, diversos estudios han reportado un abandono progresivo de la dieta mediterránea en regiones donde antiguamente era la base de la alimentación (Tur, Romaguera, y Pons, 2004; Villalón Mir et al., 2012). Esto se debe principalmente a una reducción de las cosechas agrícolas, lo que permitía mantener una dieta equilibrada de manera sencilla, mientras que, actualmente, hay una menor disponibilidad para el acceso a los productos naturales, según explican Restrepo, Morales, Ramírez, López y Varela (2006).

Para lograr revertir la tendencia de la población infanto-juvenil hacia unos índices de sobrepeso insostenibles es necesario contar con dos agentes mediadores entre dicho grupo y la adherencia a la dieta mediterránea: la familia y la escuela. Ambos entornos forman el escenario del desarrollo de la vida cotidiana de cualquier persona que no ha alcanzado la adultez y se conoce la importante influencia que ejercen sobre su estilo de vida (Dundas, Leyland y MacIntyre, 2014).

Este trabajo pretende recopilar, de forma resumida, algunas de las evidencias científicas de mayor relevancia descubiertas por investigadores familiarizados con la temática, tratando de desvelar los posibles efectos que familia y escuela poseen en la adherencia a la dieta mediterránea en poblaciones infantiles y adolescentes.

\section{Método}

\section{Diseño}

Este trabajo se trata de un estudio ex post facto en el que se ha realizado un análisis sistemático de bibliografía científica sobre la temática.

Para localizar los artículos objeto de estudio, se realizaron búsquedas en bases de datos internacionales como son: Scopus, SciELO, Web of Science y Google Scholar.

El total de documentos de carácter científico analizados fue de 49 .

En las tres primeras bases de datos se siguió el mismo procedimiento, insertando las pertinentes ecuaciones de búsqueda en español e inglés y extrayendo las referencias relevantes para el presente trabajo.

Finalmente, se buscaron artículos completos y material adicional en Google Scholar así como en páginas web institucionales o gubernamentales.

\section{Criterios de inclusión-exclusión}

Los documentos tenidos en cuenta son todos aquellos que ofrecían resultados concisos y relevantes para la temática. Se excluyeron aquellos con resultados difusos o que requerían de mayor investigación para establecer relaciones fiables.

\section{Resultados}

Para iniciar la revisión, sería adecuado clarificar la idea de dieta mediterránea (DM).

Aunque no existe una definición cerrada, este tipo de alimentación se caracteriza por la baja ingesta de grasas saturadas y carnes rojas y alto aporte de antioxidantes. En ella se da una alta ingesta de verduras, cereales integrales, frutas, frutos secos, legumbres, aceite de oliva (tanto para cocinar como consumido directamente) $\mathrm{y}$ un consumo moderado de pescado, lácteos $\mathrm{y}$ vino (Márquez-Sandoval, Bulló, Vizmanos, Casas-Agustench y Salas-Salvadó, 2008). Es un patrón alimentario tradicional común para las regiones que rodean el Mar Mediterráneo o se encuentran bajo su influencia.

En la actualidad, y según diversos estudios llevados a cabo en países bajo la influencia de este mar se han encontrado bajos o moderados niveles de adherencia a la dieta mediterránea. Concretamente en España, Grecia, Turquía, Italia, Israel, Portugal, Chipre, Alemania y otros países (Kontogianni, 2008; MariscalArcas et al., 2009 ; Noale, 2014; Peng, Goldsmith y Berry, 2017; Rodrigues, Muc, Rodrigues, Pinto y Padez, 2016; Sahingoz y Sanlier, 2011; Tognon et al., 2014; Yardimci, Ozdogan, Ozcelik y Hovland, 2016).

\section{Familia y adherencia a la dieta mediterránea}

Como primer agente socializador del niño, la familia suele poseer un papel clave en todos los aspectos de la vida de este y "es en la familia donde se adquieren los valores que permitirán analizar el mundo exterior, si bien la incorporación a otras estructuras sociales (escuelas, centros recreativos, grupos de amigos, iglesias, asociaciones, etc.) y la influencia de los medios de comunicación social (TV, Internet, ...) también contribuirán a su socialización" (Bas y Pérez de Guzmán, 2010 citado en Pallarés Piquer, 2014).

A este respecto, algunos estudios determinan, directamente, la influencia que la familia ejerce para consolidar unos hábitos alimentarios saludables, mientras que otros autores apuntan a un control incorrecto por parte de los padres, lo que conlleva a una alimentación inadecuada y una disminución de las oportunidades de auto-control (Birch y Fisher, 1998; Martínez, Llull, del Mar Bibiloni, Pons y Tur, 2010).

En la línea de la última afirmación, Agostoni et al. (2011) recomiendan a las familias que adecúen las porciones de comida a la edad y tamaño corporal del niño. Así mismo, animan a evitar consumos regulares de comida rápida o alimentos de alta densidad calórica. Animan a la promoción de tentempiés saludables y al consumo regular de comida elaborada en el seno familiar. Roccaldo et al. (2014) señalan la importancia de que los más pequeños no tengan acceso libre a la comida, sino que es más adecuado que esté supervisado por un adulto.

Según Sotos-Prieto et al. (2015), los hábitos de los niños respecto a la alimentación mediterránea y la actividad física se ven mejorados en base al nivel de concienciación sobre la salud que tengan los padres.

Además, existen algunos factores directamente relacionados con la familia que parecen influir en la adherencia de los niños. Algunos de ellos se detallan a continuación.

Respecto al núcleo de residencia de la familia, aquellos niños que viven en núcleos urbanos parecen tener una adherencia a la dieta mediterránea más pobre que los residen en áreas rurales (Grosso et al., 2013; 
Grosso y Galvano, 2016). Grao Cruces et al. (2013), realizaron un estudio en el que encontraron mayores niveles de adherencia en poblaciones rurales respecto a las urbanas, tomando 10.000 habitantes como el mínimo para considerar una población urbana.

En cuanto al nivel educativo de los padres, buena parte de las investigaciones encaminadas a descubrir las posibles relaciones existentes afirman que, a mayor nivel educativo de los padres, mayores niveles de adherencia a la DM encontrados en los niños. Antonogeorgos et al. (2013) encontraron que la adherencia a la DM está inversamente asociada al nivel de obesidad de los niños sólo en familias en las que, al menos, uno de los padres posee un nivel educativo alto, lo que no ocurre en familias en las que ambos tienen un bajo nivel educativo. Por su parte, Peng, Goldsmith y Berry (2017) encontraron, al analizar una muestra de 5.268 niños y adolescentes, que las niñas cuyas madres habían estado escolarizadas menos de 12 años poseían niveles más bajos de adherencia a la DM. Fauquet et al. (2016) encontraron resultados similares, indicando que el nivel educativo de los padres correlaciona positivamente con el grado de adherencia a los patrones alimentarios mediterráneos.

El nivel socioeconómico de la familia podría estar relacionado con el nivel de adherencia a la DM, descubriéndose que niveles bajos de ingresos están relacionados con una adherencia pobre y viceversa (Bonaccio et al., 2012).

Por último, y según indican los Ministerios de Educación y Ciencia y el de Sanidad y Consumo (2007), la alimentación saludable junto al fomento de la actividad física en las familias puede representar un punto de inflexión para detener el crecimiento de la obesidad infanto-juvenil. Algunos autores coinciden en esta afirmación, encontrando niveles más altos de adherencia a la dieta mediterránea en niños con buenos niveles de práctica de actividad física semanales, niveles bajos de sedentarismo o menor tiempo de pantalla (TV, Internet...) (Arriscado, Muros, Zabala y Dalmau, 2014; Muros, Cofre-Bolados, Arriscado, Zurita y Knox, 2017; Peng, Goldsmith y Berry, 2017).

Parece evidente, por tanto, la necesidad de concienciación por parte de las familias para que tomen parte de forma activa en esta dimensión esencial del desarrollo de los niños, puesto que ellas son el núcleo principal de socialización hasta cierta edad y las responsables de dar sustento y apoyo a los mismos.

\section{Escuela y adherencia a la dieta mediterránea}

La escuela es el segundo agente socializador del niño y, junto a la familia, "emergen como dos instituciones fundamentales para desencadenar los procesos evolutivos de las personas, actuando como propulsoras o inhibidoras de su crecimiento físico, intelectual, emocional y social" (Dessen y da Costa Polonia, 2007, p. 22).

Parece evidente, por tanto, que los aprendizajes e intervenciones que tengan lugar en la escuela serán clave a la hora de establecer patrones de salud perdurables.
Respecto a esta afirmación, diversos estudios actuales han comprobado las bajas tasas de adherencia a la DM en escuelas de países europeos, tanto para la población infantil como para los estudiantes de secundaria (Ayechu y Durá, 2010; Doménech Asensi, Sánchez Martínez y Ros Berruezo, 2015; Roccaldo et al., 2014).

En cambio, Roccaldo, Censi, D’Addezio, Berni Canani y Gennaro (2017) analizaron el impacto de la implementación de un programa de educación nutricional en 13 escuelas italianas. Mediante el cuestionario KIDMED evaluaron el nivel de adherencia a la DM, constatando un incremento significativo, principalmente, en aquellos sujetos que mejoraron la frecuencia de consumo de frutas y vegetales.

Por su parte, Pérez Gallardo, Bayona, Mingo y Rubiales (2011) confirman, mediante la aplicación de cuestionario KIDMED pre-post intervención, una mejora en los valores medios arrojados por el mismo tras la aplicación de actividades coordinadas por diversas administraciones sanitarias en dos muestras escolares de Soria (España).

Roccaldo et al. (2014) encontraron mejores niveles de adherencia a patrones alimentarios mediterráneos en aquellos escolares que cumplían alguna de las siguientes condiciones: comer en la escuela, gusto por la comida de la escuela y mayor conocimiento sobre hábitos alimenticios, entre otros.

\section{Discusión}

Los resultados obtenidos en este breve análisis de la bibliografía actual, referente al tema, arrojan una serie de conclusiones que parecen bastante claras.

En primer lugar, se advierte la alta prevalencia de índices de sobrepeso y obesidad en poblaciones infantojuveniles (OMS, 2016), relacionado en gran parte con los hábitos alimenticios de los más jóvenes.

Para lograr un cambio en esta tendencia, se hace imprescindible la participación de los dos principales entornos de los niños y adolescentes: la familia y la escuela.

Dado que las familias no pueden controlar, de forma directa, ciertos factores influyentes en el nivel de adherencia a la DM, tales como el nivel socioeconómico o el lugar de residencia, se hace imprescindible que tomen conciencia de algunas acciones fácilmente aplicables (Lazarou, Kalavana y Matalas, 2008), según se ha citado en la revisión: control de las cantidades de ingesta calórica, preparación de menús adecuados, evitar el acceso libre a la comida a cualquier hora, evitar alimentos con alto contenido en grasas saturadas e, incluso, realizar las comidas del día en familia (Costarelli, Koretsi y Georgitsogianni, 2013).

La escuela parece otro punto clave a tener en cuenta a la hora de lograr objetivos dietarios. La mejora en el conocimiento de hábitos alimenticos saludables será clave a la hora de obtener unos resultados adecuados. El comedor escolar, en los casos en que sea posible, también representa una oportunidad de establecer unas rutinas alimentarias óptimas (Roccaldo et al., 2014).

La implicación de las administraciones educativas y sanitarias mediante programas y actividades de esta 
temática han demostrado su eficacia a la hora de aumentar la adherencia a la DM (Akdemir, Donmez y Polat, 2017; Doménech Asensi, Sánchez Martínez y Ros Berruezo, 2015), por lo que se alienta a las todos los agentes implicados en la escuela para la creación de programas dirigidos a estas vertientes.

\section{Referencias}

Agostoni, C., Braegger, C., Decsi, T., Kolacek, S., Koletzko, B., Mihatsch, W., ... \& Turck, D. (2011). Role of dietary factors and food habits in the development of childhood obesity: a commentary by the ESPGHAN Committee on Nutrition. Journal of pediatric gastroenterology and nutrition, 52(6), 662669.

Akdemir, M., Donmez, L., \& Polat, H. (2017). The effect of nutritional and physical activity interventions on nutritional status and obesity in primary school children: A cluster randomized controlled study. Kuwait Medical Journal, 49(2), 105-113.

Antonogeorgos, G., Panagiotakos, D. B., Grigoropoulou, D., Papadimitriou, A., Anthracopoulos, M., Nicolaidou, P., \& Priftis, K. N. (2013). The mediating effect of parents' educational status on the association between adherence to the Mediterranean diet and childhood obesity: the PANACEA study. International journal of public health, 58(3), 401-408.

Arriscado, D., Muros, J. J., Zabala, M., \& Dalmau, J. M. (2014). Factors associated with low adherence to a Mediterranean diet in healthy children in northern Spain. Appetite, 80, 28-34.

Ayechu, A., \& Durá, T. (2010). Calidad de los hábitos alimentarios (adherencia a la dieta mediterránea) en los alumnos de educación secundaria obligatoria. Anales del sistema sanitario de Navarra, 33(1), 35-42.

Bacopoulou, F., Landis, G., Rentoumis, A., Tsitsika, A., \& Efthymiou, V. (2017). Mediterranean diet decreases adolescent waist circumference. European Journal of Clinical Investigation, 47(6), 447-455.

Bas, E., \& Pérez de Guzmán, M.V. (2010) Desafíos de la familia actual ante la escuela y las tecnologías de la información y la comunicación. EducatioXII, 28(1), 41-67.

Birch, L. L., \& Fisher, J. O. (1998). Development of eating behaviors among children and adolescents. Pediatrics, 101(Supplement 2), 539-549.

Bonaccio, M., Bonanni, A. E., Di Castelnuovo, A., De Lucia, F., Donati, M. B., de Gaetano, G., ... \& Molisani Project Investigators. (2012). Low income is associated with poor adherence to a Mediterranean diet and a higher prevalence of obesity: crosssectional results from the Moli-sani study. BMJ open, 2(6), e001685.

Costarelli, V., Koretsi, E., \& Georgitsogianni, E. (2013). Health-related quality of life of Greek adolescents: the role of the Mediterranean diet. Quality of life research, 22(5), 951-956.

Dessen, M. A., \& da Costa Polonia, A. (2007). A família e a escola como contextos de desenvolvimento humano. Paidéia, 17(36), 21-32.
Doménech Asensi, G., Sánchez Martínez, Á., \& Ros Berruezo, G. (2015). Cross-sectional study to evaluate the associated factors with differences between city and districts secondary school students of the southeast of Spain (Murcia) for their adherence to the Mediterranean diet. Nutricion hospitalaria, 31(3), 1359-1365.

Dundas, R., Leyland, A.H. \& MacIntyre, S. (2014). Early-life school, neighborhood, and family influences on adult health: A multilevel crossclassified analysis of the aberdeen children of the 1950s study. American Journal of Epidemiology, 180 (2), pp. 197-207. doi: 10.1093/aje/ kwu110

Fauquet, J., Sofi, F., López-Guimerà, G., Leiva, D., Shalà, A., Puntí, J., ... \& Casini, A. (2016). Mediterranean diet adherence among Catalonian adolescents: socio-economic and lifestyle factors. Nutrición Hospitalaria, 33(6), 1283-1290.

Grao-Cruces, A., Nuviala, A., Fernández-Martínez, A., Porcel-Gálvez, A. M., Moral-García, J. E., \& Martínez-López, E. J. (2013). Adherencia a la dieta mediterránea en adolescentes rurales y urbanos del sur de España, satisfacción con la vida, antropometría y actividades físicas y sedentarias. Nutrición Hospitalaria, 28(4), 1129-1135.

Grosso, G., \& Galvano, F. (2016). Mediterranean diet adherence in children and adolescents in southern European countries. NFS Journal, 3, 13-19.

Grosso, G., Marventano, S., Buscemi, S., Scuderi, A., Matalone, M., Platania, A., ... \& Mistretta, A. (2013). Factors associated with adherence to the Mediterranean diet among adolescents living in Sicily, Southern Italy. Nutrients, 5(12), 4908-4923.

Kindblom, J. M., Lorentzon, M., Hellqvist, Å., Lönn, L., Brandberg, J., Nilsson, S., ...Ohlsson, C. (2009). BMI Changes During Childhood and Adolescence as Predictors of Amount of Adult Subcutaneous and Visceral Adipose Tissue in Men: The GOOD Study. Diabetes, 58(4), 867-874. http://doi.org/10.2337/db08-0606

Kontogianni, M. D., Vidra, N., Farmaki, A. E., Koinaki, S., Belogianni, K., Sofrona, S., ... \& Yannakoulia, M. (2008). Adherence rates to the Mediterranean diet are low in a representative sample of Greek children and adolescents. The Journal of nutrition, 138(10), 19511956.

Lazarou, C., Kalavana, T., \& Matalas, A. L. (2008). The influence of parents' dietary beliefs and behaviours on children's dietary beliefs and behaviours. The CYKIDS study. Appetite, 51(3), 690-696.

Liria, R. (2012). Consecuencias de la obesidad en el niño y el adolescente: un problema que requiere atención. Revista Peruana de Medicina Experimental y Salud Pública, 29(3), 357-360.

Mariscal-Arcas, M., Rivas, A., Velasco, J., Ortega, M., Caballero, A. M., \& Olea-Serrano, F. (2009). Evaluation of the Mediterranean Diet Quality Index (KIDMED) in children and adolescents in Southern Spain. Public health nutrition, 12(9), 1408-1412.

Márquez-Sandoval, F., Bulló, M., Vizmanos, B., CasasAgustench, P., \& Salas-Salvadó, J. (2008). Un patrón 
de alimentación saludable: la dieta mediterránea tradicional. Antropo, 16, 11-22.

Martínez, E., Llull, R., del Mar Bibiloni, M., Pons, A., $\&$ Tur, J. A. (2010). Adherence to the Mediterranean dietary pattern among Balearic Islands adolescents. British journal of nutrition, 103(11), 1657-1664.

Menéndez García, R. A., \& Franco Díez, F. J. (2009). Publicidad y alimentación: influencia de los anuncios gráficos en las pautas alimentarias de infancia y adolescencia. Nutrición Hospitalaria, 24(3), 318-325.

Ministerio de Educación y Ciencia y Ministerio de Sanidad y Consumo . (2007). Alimentación Saludable. Guía para las familias. Madrid: Secretaría geneal técnica del MEC.

Muros, J. J., Cofre-Bolados, C., Arriscado, D., Zurita, F., \& Knox, E. (2017). Mediterranean diet adherence is associated with lifestyle, physical fitness, and mental wellness among $10-y-o l d s$ in Chile. Nutrition, $35,87-92$.

Noale, M., Nardi, M., Limongi, F., Siviero, P., Caregaro, L., Crepaldi, G., ... \& Mediterranean Diet Foundation Study Group. (2014). Adolescents in southern regions of Italy adhere to the Mediterranean diet more than those in the northern regions. Nutrition Research, 34(9), 771-779.

Ohlsson, C., Bygdell, M., Sondén, A., Rosengren, A., \& Kindblom, J. M. (2016). Association between excessive BMI increase during puberty and risk of cardiovascular mortality in adult men: a populationbased cohort study. The Lancet Diabetes \& Endocrinology, 4(12), 1017-1024.

Organización Mundial de la Salud (2016). Informe de la comisión para acabar con la obesidad infantil. Ginebra: Organización Mundial de la Salud.

Organización Mundial de la Salud. (2017). Estrategia mundial sobre régimen alimentario, actividad física y salud [online]. Recuperado de: http://www.who.int/dietphysicalactivity/pa/es/

Ozen, A. E., del Mar Bibiloni, M., Murcia, M. A., Pons, A., \& Tur, J. A. (2015). Adherence to the Mediterranean diet and consumption of functional foods among the Balearic Islands' adolescent population. Public health nutrition, 18(4), 659-668.

Pallarés Piquer, M. (2014). Medios de comunicación:i espacio para el ocio o agentes de socialización en la adolescencia?. Pedagogía Social. Revista Interuniversitaria, (23), 231-252.

Papadaki, S., \& Mavrikaki, E. (2015). Greek adolescents and the Mediterranean diet: factors affecting quality and adherence. Nutrition, 31(2), 345349.

Peng, W., Goldsmith, R., \& Berry, E. M. (2017). Demographic and lifestyle factors associated with adherence to the Mediterranean diet in relation to overweight/obesity among Israeli adolescents: findings from the Mabat Israeli national youth health and nutrition survey. Public health nutrition, 20(5), 883-892.

Pérez Gallardo, L., Bayona, I., Mingo, T., \& Rubiales, C. (2011). Utilidad de los programas de educación nutricional para prevenir la obesidad infantil a través de un estudio piloto en Soria. Nutrición Hospitalaria, 26(5), 1161-1167.

Restrepo, S. L., Morales, R. M., Ramírez, M. C., López, M. V., \& Varela, L. E. (2006). Los hábitos alimentarios en el adulto mayor y su relación con los procesos protectores y deteriorantes en salud. Revista chilena de nutrición, 33(3), 500-510.

Rice, F. P. (1997). Desarrollo humano: estudio del ciclo vital. Mexico: Pearson Educación.

Roales Nieto, J. G., Moreno San Pedro, E., Vinaccia, S., Contreras, F., Fernández, H., Londoño, X., ... \& Medellín Moreno, J. (2004). Hábitos básicos de salud y creencias sobre salud y enfermedad en adolescentes de España, Colombia y México. Revista Latinoamericana de Psicología, 36(3), 483-504.

Roccaldo, R., Censi, L., D'Addezio, L., Berni Canani, S., \& Gennaro, L. (2017). A teachers' training program accompanying the "School Fruit Scheme" fruit distribution improves children's adherence to the Mediterranean diet: an Italian trial. International Journal of Food Sciences and Nutrition, 1-14.

Roccaldo, R., Censi, L., D’Addezio, L., Toti, E., Martone, D., D'Addesa, D., ... \& D'Amicis, A. (2014). Adherence to the Mediterranean diet in Italian school children (The ZOOM8 Study). International journal of food sciences and nutrition, 65(5), 621-628.

Rodrigues, D., Muc, M., Rodrigues, P. R. M., Pinto, A. M., \& Padez, C. (2016). Dietary patterns and their socioeconomic and behavioral determinants in 6-to 8Year-Old Portuguese children. Ecology of food and nutrition, 55(5), 428-441.

Sahingoz, S. A., \& Sanlier, N. (2011). Compliance with Mediterranean Diet Quality Index (KIDMED) and nutrition knowledge levels in adolescents. A case study from Turkey. Appetite, 57(1), 272-277.

Sotos-Prieto, M., Santos-Beneit, G., Pocock, S., Redondo, J., Fuster, V., \& Peñalvo, J. L. (2015). Parental and self-reported dietary and physical activity habits in pre-school children and their socioeconomic determinants. Public health nutrition, 18(2), 275-285.

Subcomisión de Epidemiología, \& Comisión de Nutrición. (2005). Consenso sobre factores de riesgo de enfermedad cardiovascular en pediatría. Obesidad. Arch. argent. pediatr, 103(3), 262.

Tognon, G., Hebestreit, A., Lanfer, A., Moreno, L. A., Pala, V., Siani, A., ... \& Ahrens, W. (2014). Mediterranean diet, overweight and body composition in children from eight European countries: crosssectional and prospective results from the IDEFICS study. Nutrition, Metabolism and Cardiovascular Diseases, 24(2), 205-213.

Tur, J. A., Romaguera, D., \&amp; Pons, A. (2004). Food consumption patterns in a Mediterranean region: does the Mediterranean diet still exist?. Annals of Nutrition and Metabolism, 48(3), 193-201.

Villalón Mir, M., Cutillas, L., Martínez-Martínez, F., López García de la Serrana, H., Oliveras-López, M. J., \& Samaniego-Sánchez, C. (2012). La Oficina de Farmacia: una herramienta para conocer el grado de adherencia a la dieta mediterránea de la población. 
Yardimci, H., Ozdogan, Y., Ozcelik, A.O., \& Hovland, E.D. (2016). Turkish adolescents' adherence to the mediterranean diet. Anthropologist, 25(1-2), 174-179. 